\title{
ADAM17 promotes epithelial-mesenchymal transition via TGF- $\beta$ /Smad pathway in gastric carcinoma cells
}

\author{
MIN XU ${ }^{1 *}$, HAILANG ZHOU $^{1 *}$, CHUNLI ZHANG $^{1}$, JUNBO HE $^{1}, \mathrm{HONG} \mathrm{WEI}^{1}$, MENG ZHOU $^{1}$, YING LU $^{1}$, \\ YAOCHENG SUN ${ }^{1}$, JERRY WANMING DING ${ }^{3}$, JIAN ZENG ${ }^{2}$, WANXIN PENG ${ }^{2}$, FENGYI DU $^{2}$ and AIHUA GONG ${ }^{2}$ \\ ${ }^{1}$ Department of Gastroenterology, Affiliated Hospital of Jiangsu University, Jiangsu University, Zhenjiang, Jiangsu; \\ ${ }^{2}$ Department of Cell Biology, School of Medicine, Jiangsu University, Zhenjiang, Jiangsu, P.R. China; \\ ${ }^{3}$ Department of Physiology and Pharmacology, Schulich School of Medicine and Dentistry, \\ The University of Western Ontario, London, ON, N6A 5C1, Canada
}

Received July 23, 2016; Accepted October 11, 2016

DOI: $10.3892 /$ ijo.2016.3744

\begin{abstract}
Although a disintegrin and metalloproteinase-17 (ADAM17) overexpression has been demonstrated in numerous human tumors including gastric cancer, its role in gastric cancer development remains to be clarified. In the present study, we identify that ADAM17 activates TGF- $\beta /$ Smad signaling to promote epithelial-mesenchymal transition (EMT) in gastric cancer cells. We found that ADAM17 promotes proliferation, migration and invasion in gastric carcinoma cells. Subsequently, we revealed that silencing ADAM17 induces the expression of epithelial marker of E-cadherin and downregulates expression of mesenchymal markers including $\mathrm{N}$-cadherin, vimentin and Snail in MGC803 and MKN45 cells, whereas ADAM17 overexpression reverses these changes in BGC823 and HGC27 cells. Furthermore, ADAM17 knockdown significantly inhibits the expression of TGF- $\beta$ and its downstream signaling molecules p-Smad2 and p-Smad3 in MGC803 and MKN45 cells. Consistently, ADAM17 overexpression reversed these changes in BGC823 and HGC27 cells. These results suggest that ADAM17 promotes epithelial-mesenchymal transition via the TGF- $\beta /$ Smad pathway. Collectively, the present study demonstrates that ADAM17 plays a critical role in the development of gastric cancer and provides a potential therapeutic target for gastric cancer.
\end{abstract}

\section{Introduction}

Gastric cancer is the fourth most commonly diagnosed cancer and the second leading cause of cancer death world-

Correspondence to: Professor Aihua Gong, Department of Cell Biology, School of Medicine, Jiangsu University, 301 Xuefu Road, Zhenjiang, Jiangsu 212013, P.R. China

E-mail: ahg5@mail.ujs.edu.cn

${ }^{*}$ Contributed equally

Key words: ADAM17, epithelial-mesenchymal transition, gastric carcinoma cells, migration and invasion, TGF- $\beta /$ Smad signaling wide, especially prevalent in developing countries (1). The only potentially curative treatment for gastric cancer is complete resection (2). However, despite aggressive surgical intervention, more than $50 \%$ of patients undergoing radical resection will experience disease recurrence, usually in the form of metastatic disease (3). Thus, a better understanding of the underlying mechanisms that promote pathogenesis and progression of gastric cancer is urgently needed.

ADAM17, as a sheddase, releases extracellular domains of transmembrane proteins, which thereby modulate cell-cell and cell environment communication (4). ADAM17 overexpression has been demonstrated in numerous human tumors including gastric cancer (5), and several well-designed studies have shown correlations between the levels of ADAM17 expression and tumor progression $(6,7)$. A previous study found that the expression levels of ADAM17 mRNA and protein in gastric cancer tissues are both significantly higher than those in non-cancerous gastric mucosa (8). It is also identified that the siRNA-targeted ADAM17 transcripts suppress deoxycholate (DC)-induced activation of EGFR and ERK1/2, suggesting that in AGS human gastric cancer cells, DC transactivates EGFR through M-BAR- and ADAM/HB-EGF-dependent mechanisms (9). Another study reported that ADAM17 activated by TGF- $\beta$ mediates proHB-EGF shedding to promote the proliferation of gastric cancer cells via EGFR transactivation (10). According to current views, the major mechanism by which ADAM17 supports cancer development involves shedding, and thus, activation of growth factors such as TGF- $\alpha$, HB-EGF, amphiregulin or neuregulins $(11,12)$. In turn, these growth factors stimulate survival, proliferation and migration of tumor cells. Therefore, ADAM17 may be an important molecular marker for predicting carcinogenesis, progression and prognosis of gastric cancer.

It is reported that epithelial-mesenchymal transition (EMT) plays a critical role in the cancer progression and metastasis, including gastric cancer $(13,14)$. EMT is a process characterized by loss of cell-cell adhesion and increase of cell motility (15). During EMT, significant morphological transformation occurs, including reduced expression of epithelial markers, such as E-cadherin, and increased expression of mesenchymal markers, such as $\mathrm{N}$-cadherin and vimentin 
Table I. The primer sequences for the GAPDH, ADAM17, MMP-2 and MMP-9 genes used in real-time PCR experiments.

Forward primer
Reverse primer

$\begin{array}{ll}\text { GAPDH } & \text { 5'-GGTGAAGGTCGGTGTGAACG-3' } \\ \text { ADAM17 } & \text { 5'-AGAGCTGACCCAGATCCCAT-3' } \\ \text { MMP-2 } & \text { 5'-CACAGGAGGAGAAGGCTGTG-3' } \\ \text { MMP-9 } & \text { 5'-TTCAGGGAGACGCCCATTTC-3' }\end{array}$

5'-CTCGCTCCTGGAAGATGGTG-3'

5'-TACTCTCTTCCCCTCTGCCC-3'

5'-GAGCTTGGGAAAGCCAGGAT-3' 5'-TGTAGAGTCTCTCGCTGGGG-3'
$(16,17)$. However, there are few reports concerning the association between ADAM17 expression and EMT in gastric cancer.

In the present study, we examined the roles of ADAM17 in EMT of gastric carcinoma cells and elucidated the underlying mechanism. Our data show that ADAM17 promotes the proliferation, migration and invasion of gastric carcinoma cells. Importantly, ADAM17 promotes EMT probably via TGF- $\beta /$ Smad signaling in gastric carcinoma cells.

\section{Materials and methods}

Cell culture. The gastric carcinoma cell lines MGC803, MKN45, HGC27 and BGC823 were purchased from the Type Culture Collection of the Chinese Academy of Sciences (Shanghai, China). The cells were cultured with Dulbecco's modified Eagle's medium (DMEM; HyClone Laboratories, Beijing, China) supplemented with $10 \%$ fetal bovine serum (FBS; Gibco, Carlsbad, CA, USA), in humidified $5 \% \mathrm{CO}_{2}$ incubator at $37^{\circ} \mathrm{C}$.

Real-time PCR. Total RNA was isolated using RNAiso Plus (Takara Bio, Shiga, Japan). Reverse transcription was performed using RevertAid First Strand cDNA Synthesis kit (Thermo Fisher Scientific) according to the manufacturer's recommendations. The SYBR-Green-based real-time PCR was then performed in triplicate using CFX-96 sequence detection system (Bio-Rad Laboratories) and gene expression was normalized by GAPDH. Primers are listed in Table I. The relative fold change in RNA expression was calculated using the $2^{-\Delta \Delta \mathrm{Ct}}$ method.

Plasmid construction. The ADAM17 shRNA sequence was obtained from Sigma Company official website, which was produced by Sangon Biotech, Co., Ltd. (Shanghai, China). The oligo sequence of ADAM17 shRNA included: ADAM17 shRNA (F): 5'-CCG GCC TAT GTC GAT GCT GAA CAA ACT CGA GTT TGT TCA GCA TCG ACA TAGG TTT TTG-3' and ADAM17 shRNA (R): 5'-AAT TCA AAA ACC TAT GTC GAT GCT GAA CAA ACT CGA GTT TGT TCA GCA TCG ACA TAG G-3'. The ADAM17 shRNA sequence was inserted into the EcoRI and AgeI site of the pLKO.1-TRC plasmid and ligated into the vector (Sigma-Aldrich, St. Louis, MO, USA).

Lentivirus production and cell transduction. The packaging plasmid psPAX2 and the envelope plasmid pMD2.G were purchased from Sigma-Aldrich. PLKO.1-sh-ADAM17 was cotransfected with psPAX2 and pMD2.G into HEK293T cells using Lipofectamine 2000 (Invitrogen, Carlsbad, CA, USA).
Viruses were harvested $48 \mathrm{~h}$ after transfection and viral titers were determined. Cells were infected with $1 \times 10^{6}$ recombinant lentivirus transduction units in the presence of $8 \mathrm{mg} /$ $\mathrm{ml}$ polybrene Sigma-Aldrich. Puromycin (1:10,000 dilutions) was added to cells until the cells in the blank group were nonviable. Cells which survived were stable infected cells.

Transient transfection. Cells were seeded in 6-well plates at a density of $4 \times 10^{5}$ cells/well. After $24 \mathrm{~h}$ of culture, the medium was replaced by Opti-MEM (Invitrogen) and cultured. In total, $2 \mu \mathrm{g}$ plasmid was transfected using $6 \mu \mathrm{l}$ Lipofectamine 2000 transfection reagent (Invitrogen). After incubation for another $48 \mathrm{~h}$, the treated cells were used to investigate the effect of gene rescue using western blot analysis or Transwell and Cell Counting kit-8 assay.

Western blotting. The cultured cells were rinsed with cold phosphate-buffered saline (PBS) before treated with RIPA lysis buffer at $4^{\circ} \mathrm{C}$ for $10 \mathrm{~min}$. Then the mixture was centrifuged under $4^{\circ} \mathrm{C}$ at $12,000 \mathrm{r} / \mathrm{min}$ for $15 \mathrm{~min}$. The supernatant was removed and the protein concentration was measured with the BCA method. Approximately $40 \mu \mathrm{g}$ of protein was loaded in each lane, and separated by $10 \%$ SDS-PAGE and then transferred to the PVDF membrane. The membrane was blocked by $5 \%$ non-fat milk powder for $1 \mathrm{~h}$ at room temperature before overnight incubation with primary antibodies $4^{\circ} \mathrm{C}$, followed by the secondary antibody. The antibodies were rabbit antiADAM17 (cat. no. 3976), mouse anti- $\beta$-tubulin (cat. no. 6181), rabbit anti-N-cadherin (cat. no. 13116), rabbit anti-E-cadherin (cat. no. 3195), rabbit anti-vimentin (cat. no. 5741), rabbit antiSnail (cat. no. 3879), rabbit anti-TGF- $\beta$ (cat. no. 3711), rabbit anti-Smad2 (cat. no. 5339), rabbit anti-p-Smad2 (cat. no. 3108), rabbit anti-Smad3 (cat. no. 9523), rabbit anti-p-Smad3 (cat. no. 9520) (all from Cell Signaling Technology, Danvers, MA, USA).

Cell Counting kit-8 assay. The measurement of viable cell mass was performed with Cell Counting kit-8 (Beyotime Institute of Biotechnology, Shanghai China) according to the manufacturer's instructions. Briefly, 3,000 cells/well were seeded in a 96-well plate, grown in an incubator $\left(5 \% \mathrm{CO}_{2}\right.$, at $\left.37^{\circ} \mathrm{C}\right)$. Respectively in the first, second, third, fourth and fifth day, $10 \mu$ l CCK-8 was added to each well, and cells were incubated at $37^{\circ} \mathrm{C}$ for $2 \mathrm{~h}$ and the absorbance was finally determined at $490 \mathrm{~nm}$.

Colony-forming assay. Transfected MGC803 and MKN45 cells were harvested, resuspended in medium and transferred to the 6-well plate (500, 1,000 and 2,000 cells/well) for 10-14 days until large colonies were visible. Colonies were fixed and 
A
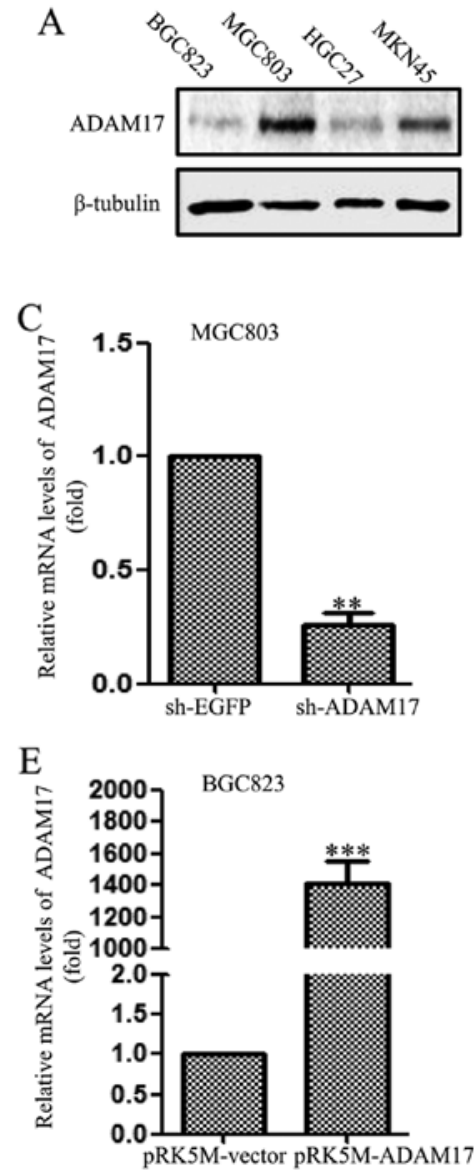
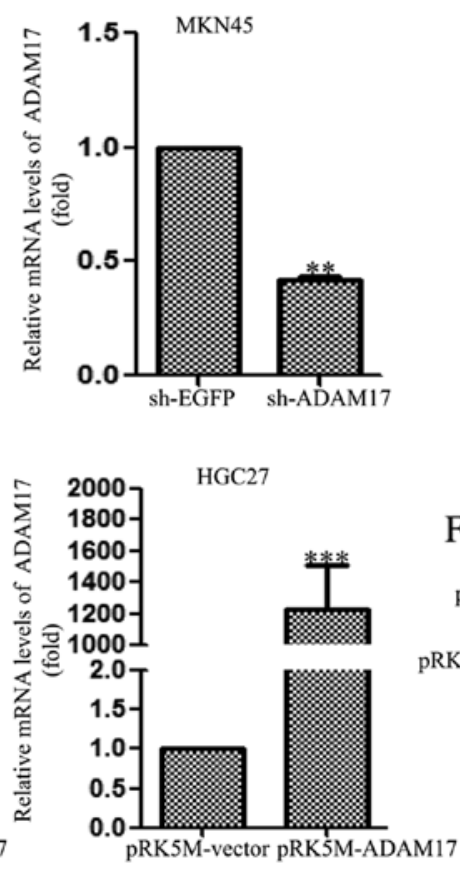

$\mathrm{B}$
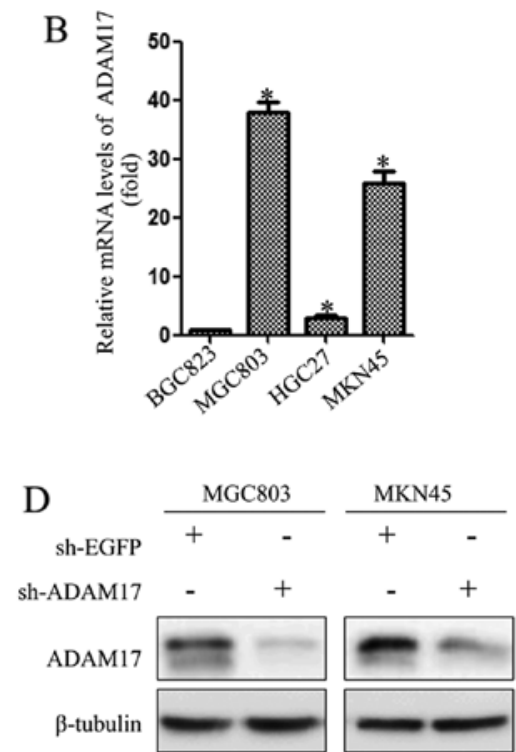

$\mathrm{F}$
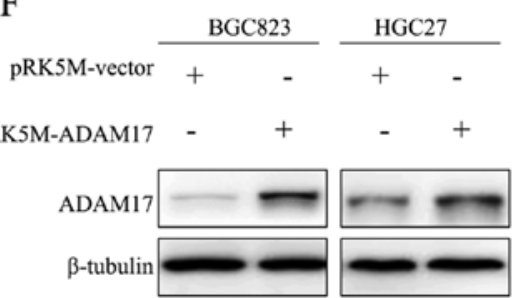

Figure 1. ADAM17 expression in different gastric carcinoma cells. (A and B) Relative expression levels of ADAM17 protein and mRNA were assessed in MGC803, MKN45, BGC823 and HGC27 cells. "P<0.05. (C) Identification of shRNA-mediated knockdown of ADAM17 in MGC803 and MKN45 cells by real-time PCR analysis. GAPDH was used for normalization (Student's t-test, ${ }^{* *} \mathrm{P}<0.001$ vs. sh-EGFP). (D) Identification of shRNA-mediated knockdown of ADAM17 in MGC803 and MKN45 cells by western blot analysis. (E) Identification of ADAM17 overexpression in BGC823 and HGC27 cells by real-time PCR analysis. GAPDH was used for normalization (Student's t-test, ${ }^{* * *} \mathrm{P}<0.001$ vs. sh-EGFP). (F) Identification of ADAM17 overexpression in BGC823 and HGC27 cells by western blot analysis.

stained with $0.05 \%$ crystal violet for $30 \mathrm{~min}$, and the number of colonies was counted or photomicrographs were taken under phase-contrast microscope.

Wound healing assay. Cells have grown to confluence in complete cell culture medium. At time $0 \mathrm{~h}$, a scrape wound was created across the diameter with a $10-\mu 1$ pipette tip followed by extensive washes with medium to remove dead and floating cells. The distance was recorded at 0 and $48 \mathrm{~h}$. Images were captured using an inverted microscope equipped with a digital camera.

Migration assay and invasion assay. For assessing cell migration, $1 \times 10^{5}$ cells in serum-free media were seeded into the Transwell inserts (Corning) containing $8-\mu \mathrm{m}$ permeable pores and were allowed to migrate toward $10 \%$ FBS-containing medium. Twenty-four to $36 \mathrm{~h}$ later, the migrated cells on the bottom of the insert were fixed with $4 \%$ paraformaldehyde solution followed by crystal violet (1\%) staining. Images were taken after washing the inserts three times with PBS. Five independent fields were counted for each Transwell and the average numbers of cells/field were represented as graphs. For assessing cell invasion, $1 \times 10^{5}$ cells in serum-free medium were seeded in the Transwell inserts which had been covered with a layer of BD Matrigel basement membrane. The cells were later processed similarly to that of cell migration assay. Finally, invaded cells were counted and the relative number was calculated.

Statistical analysis. The data are presented as mean \pm SD from at least three independent experiments. All statistical analyses were carried out using SPSS Statistics 19 software. Comparisons between the groups were analyzed using the Student's t-test (two groups) or a one-way ANOVA (multiple groups). $\mathrm{P}<0.05$ was considered statistically significant.

\section{Results}

ADAM17 expression in gastric carcinoma cells. First, we analyzed the ADAM17 expression in MGC803, MKN45, HGC27 and BGC823 cells using real-time PCR and western blot analysis. We found that ADAM17 expression, at both mRNA and protein levels, was higher in MGC803 and MKN45 cells than that in HGC27 and BGC823 cells (Fig. 1A and B). Subsequently, we constructed plasmids sh-ADAM17 and pRK5M-ADAM17 to identify the role of ADAM17 in the development of gastric cancer, and then we examined the knockdown effect of sh-ADAM17 at both 


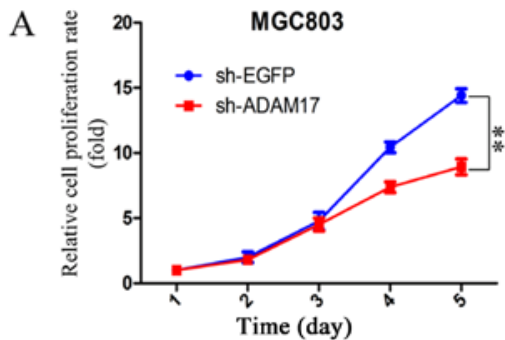

B

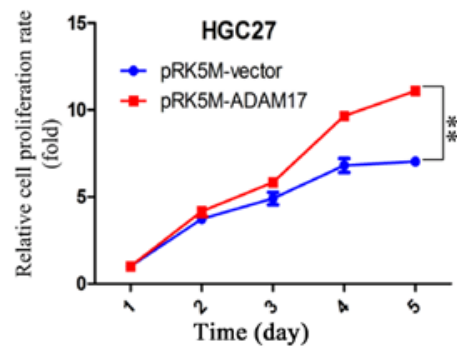

C

MGC803

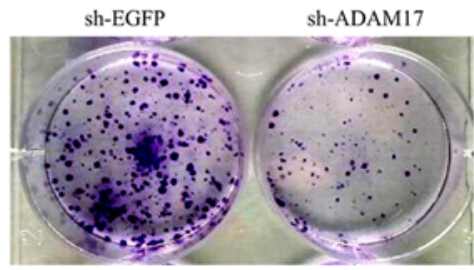

sh-EGFP

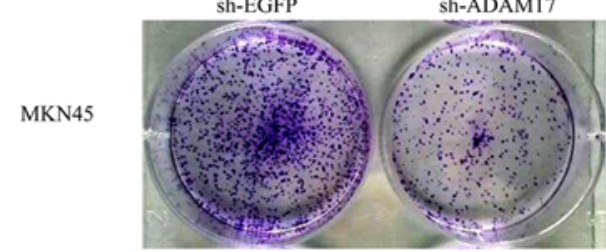

MKN45

$\mathrm{D}$
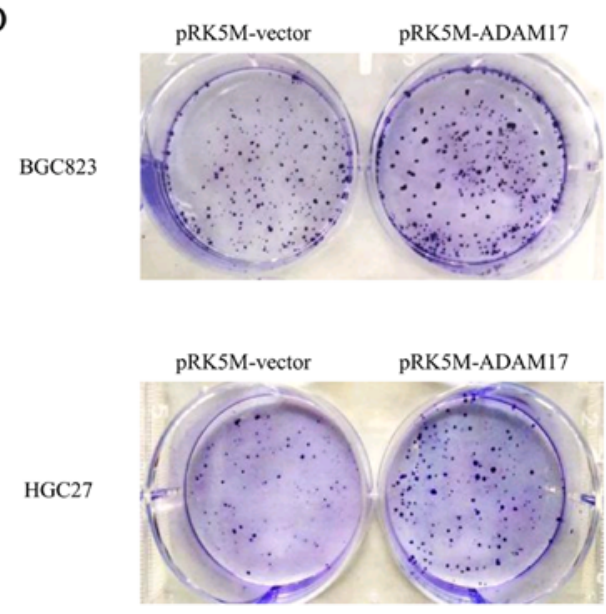
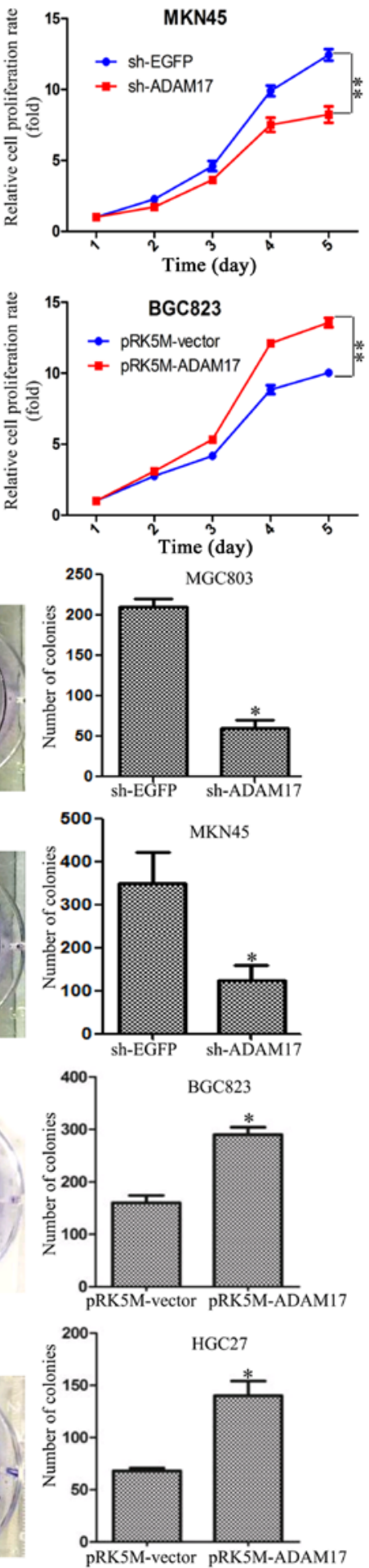

Figure 2. ADAM17 promotes cell proliferation and colony formation in gastric carcinoma cells. (A) CCK-8 assay showed that ADAM17 knockdown inhibited MGC803 and MKN45 cell growth (Student's t-test, ${ }^{* *} \mathrm{P}<0.001$ vs. sh-EGFP). (B) CCK-8 assay showed that ADAM17 overexpression promoted BGC823 and HGC27 cell growth (Student's t-test, ${ }^{* *} \mathrm{P}<0.001$ vs. pRK5M-vector). (C) Clone formation assays in MGC803 and MKN45 cells. ADAM17 knockdown inhibited cell clone formation (Student's t-test, "P<0.05 vs. sh-EGFP). (D) Clone formation assays in BGC823 and HGC27 cells. ADAM17 overexpression promoted cell clone formation (Student's t-test, ${ }^{\mathrm{P}}<0.05$ vs. pRK5M-vector).

protein and mRNA levels using sh-EGFP as a control. The mRNA and protein levels of ADAM17 were significantly decreased in sh-ADAM17 group compared with sh-EGFP group (Fig. 1C and D). Similarly, pRK5M-vector or pRK5MADAM17 were transferred into HGC27 and BGC823 cells, then ADAM17 mRNA and protein levels were examined by real-time PCR and western blot analysis, and the results indicated that ADAM17 expression at both mRNA and protein levels was significantly increased in pRK5M-ADAM17 group compared with pRK5M-vector group (Fig. 1E and F).

ADAM17 promotes proliferation and colony formation in gastric carcinoma cells. To investigate the effect of ADAM17 on cell growth in gastric carcinoma cells, we first used the 

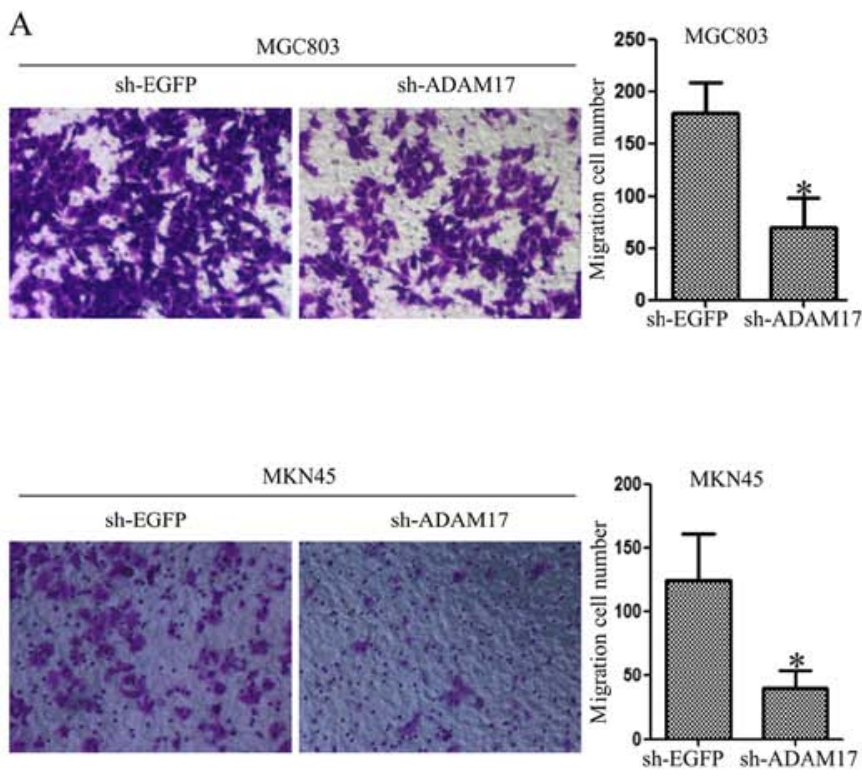

$\mathrm{C}$
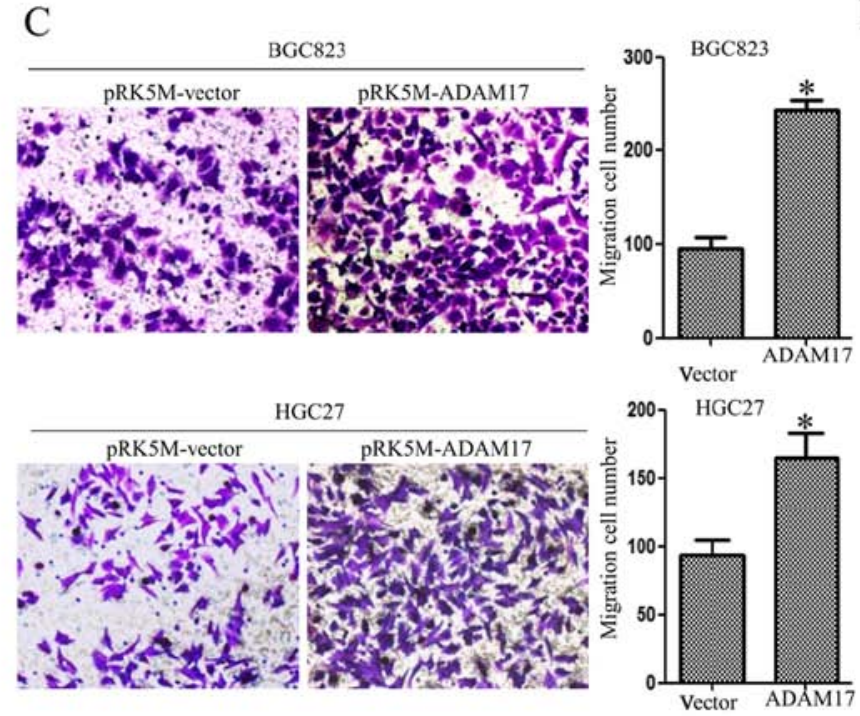

D
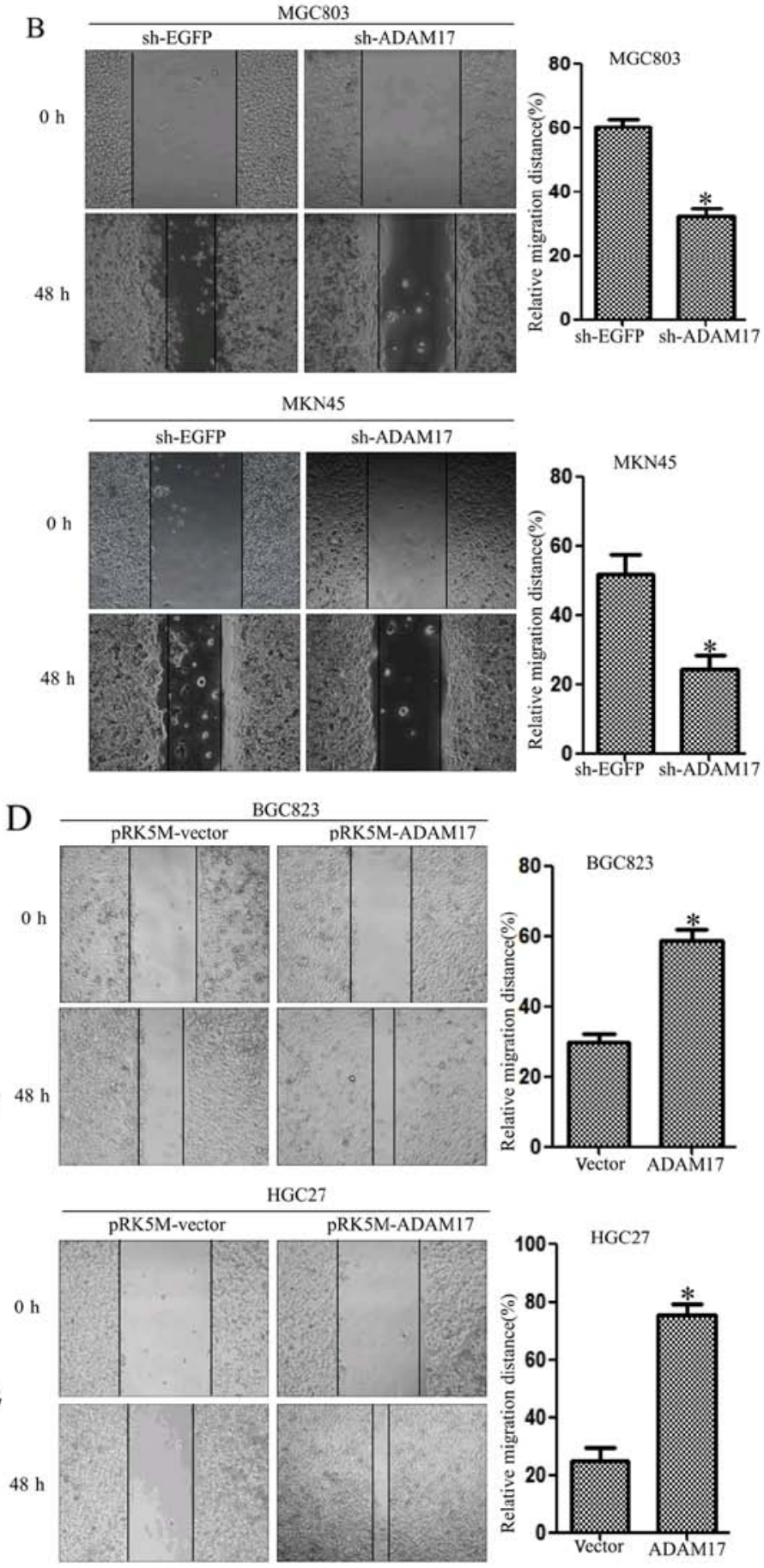

Figure 3. ADAM17 promotes cell migration in gastric carcinoma cells. (A and B) The migration change was measured by Transwell assay and wound scratch assay in MGC803 and MKN45 sh-ADAM17 stably infected cells (Student's t-test, ${ }^{*} \mathrm{P}<0.05$ vs. sh-EGFP). (C and D) After transfected with pRK5M-vector or pRK5M-ADAM17 plasmid in BGC823 and HGC27 cells, the migration change was measured by Transwell assay and wound scratch assay (Student's t-test, *P<0.05 vs. pRK5M-vector).

CCK-8 assay to determine the growth curves. The results indicated that ADAM17 knockdown significantly inhibited the proliferation of MGC803 and MKN45 cells (Fig. 2A). Expectedly, overexpression of ADAM17 promotes the ability of proliferation in HGC27 and BGC823 cells (Fig. 2B). To further confirm the effect of ADAM17 on proliferation of gastric carcinoma cells, we evaluated their ability of colony formation in the above mentioned cells. Colonies with strong, highly dense staining and at least 50 cells per colony were counted. We found that ADAM17 knockdown resulted in smaller colonies and lower colony density compared to the control group in both MGC803 and MKN45 cells. The colony formation rates were $202 \pm 11$ and $50 \pm 9$ in sh-EGFP and sh-ADAM17 MGC803 cells, and $331 \pm 8$ and $114 \pm 7$ in sh-EGFP and sh-ADAM17 MKN45 cells (Fig. 2C). To confirm the above results, the HGC27 and BGC823 cells were transfected with pRK5M-vector or pRK5M-ADAM17 plasmids, respectively. We found that ADAM17 overexpression increased the colony sizes and densities compare to control group, and the number of colonies (defined as $\geq 50$ cells) was $150 \pm 10$ and $300 \pm 13$ in 
A
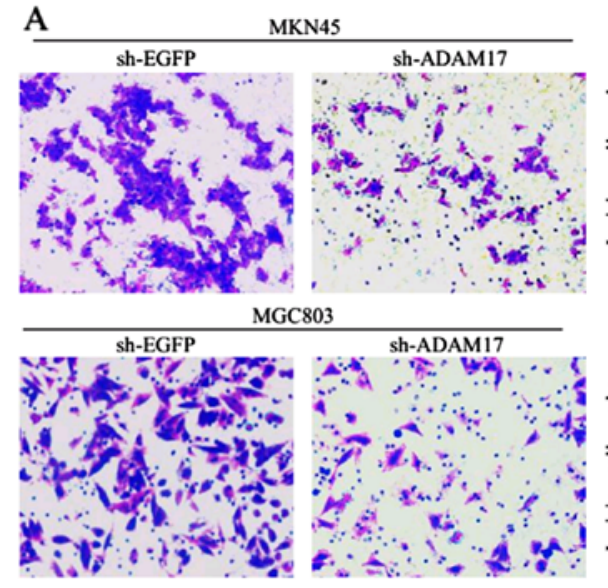

C

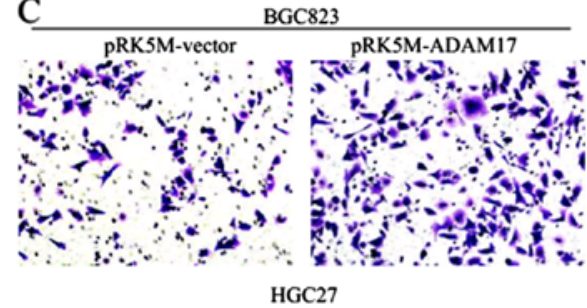
HGC27

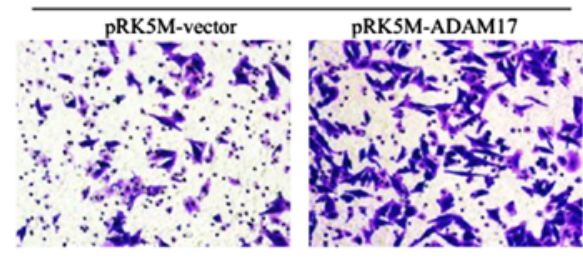

E
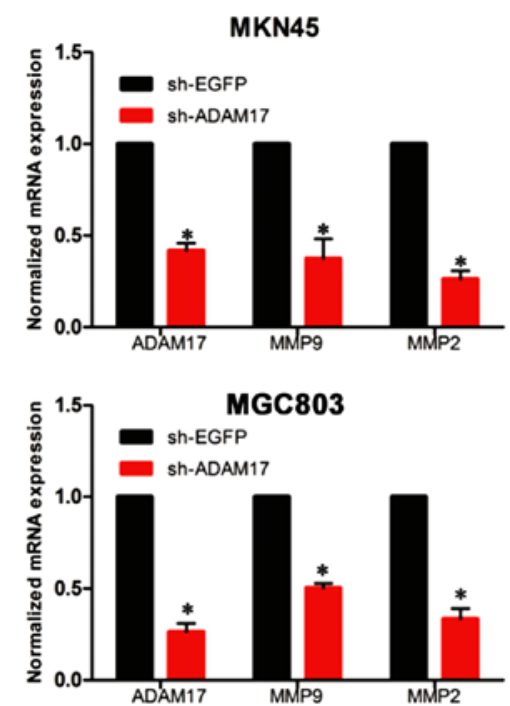

B
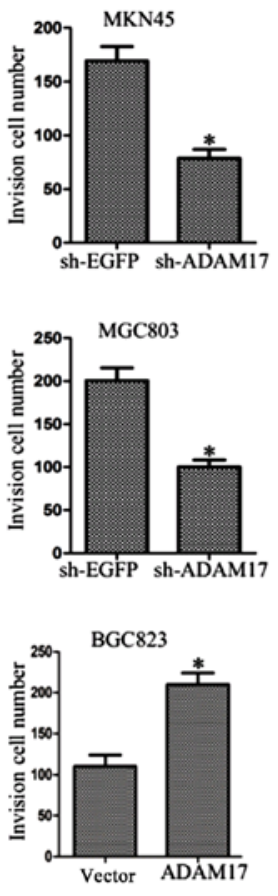

D
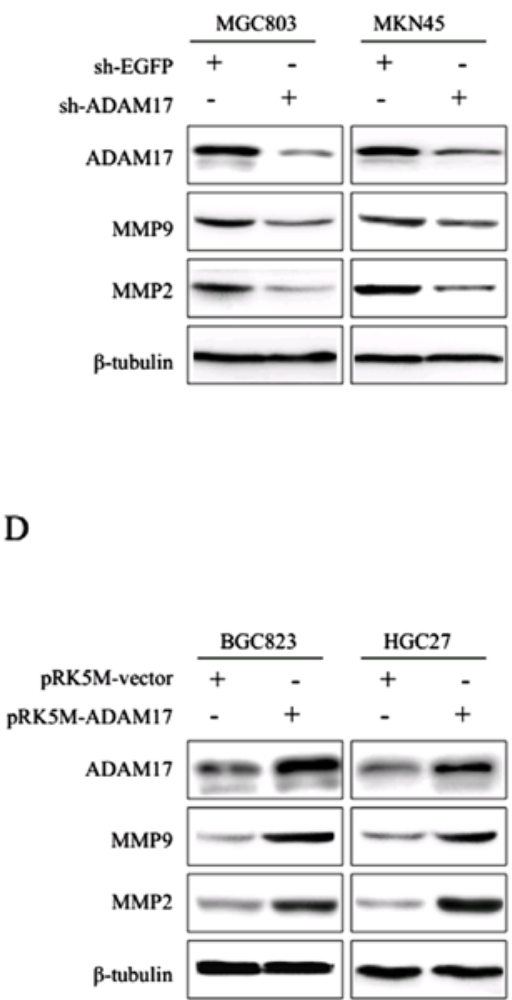

$\mathrm{F}$
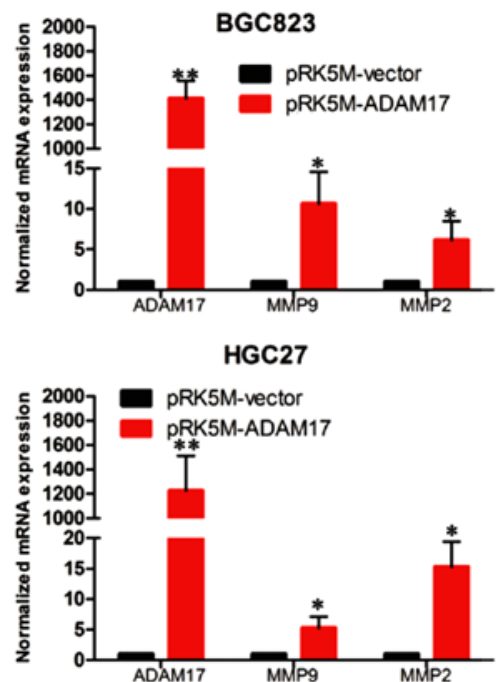

Figure 4. ADAM17 promotes cell invasion in gastric carcinoma cells. (A) The invasion changes were measured by Transwell assay in MGC803 and MKN45 sh-ADAM17 stable infected cells (Student's t-test, ${ }^{*} \mathrm{P}<0.05$ vs. sh-EGFP). (C) After transfected with pRK5M-vector or pRK5M-ADAM17 plasmid in BGC823 and HGC27 cells, the invasion change was measured by Transwell assay and wound scratch assay (Student's t-test, ${ }^{*} \mathrm{P}<0.05$ vs. pRK5M-vector). (B and D) The protein levels of MMP-2 and MMP-9 were measured by western blot analysis. (E and F) The mRNA levels of MMP-2 and MMP-9 were measured by real-time PCR (Student's t-test, $\left.{ }^{*} \mathrm{P}<0.05,{ }^{* *} \mathrm{P}<0.001\right)$.

BGC823 cells, $77 \pm 12$ and $153 \pm 9$ in HGC27 cells, respectively (Fig. 2D). It was confirmed that ADAM17 promotes proliferation and colony formation in gastric carcinoma cells.

ADAM17 enhances the migration ability of gastric carcinoma cells. Next, we examined the ability of migration by Transwell assays and wound scratch assays. First, we examined the migratory potential of MGC803 and MKN45 cells using Transwell assays. We found that the migration rates were $180 \pm 15$ and $60 \pm 12$ in sh-EGFP and sh-ADAM17 MGC803 cells, and $120 \pm 14$ and $40 \pm 11$ in sh-EGFP and sh-ADAM17 MKN45 cells (Fig. 3A). To test the effects of ADAM17 knockdown on cell motility, we performed wound scratch assays. For these assays, a scrape wound was created on confluent cultures of MGC803 
A

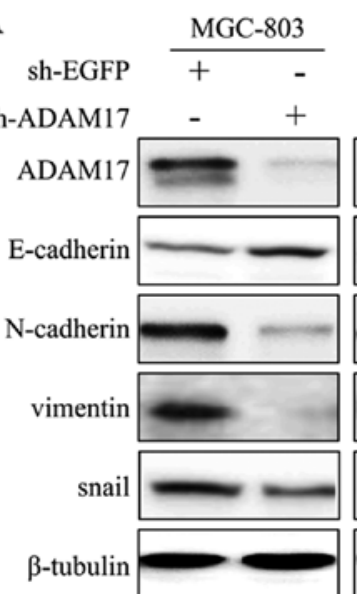

C

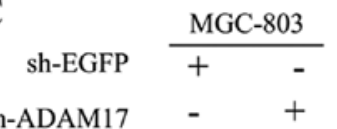

sh-ADAM17 - $\quad+$

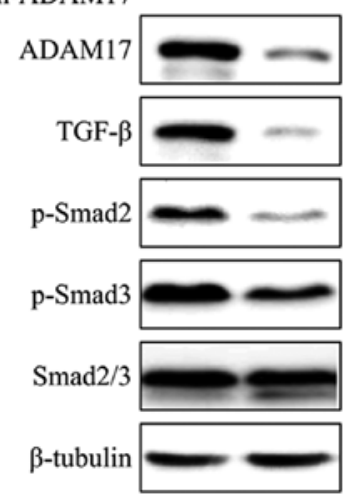

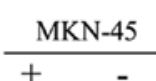
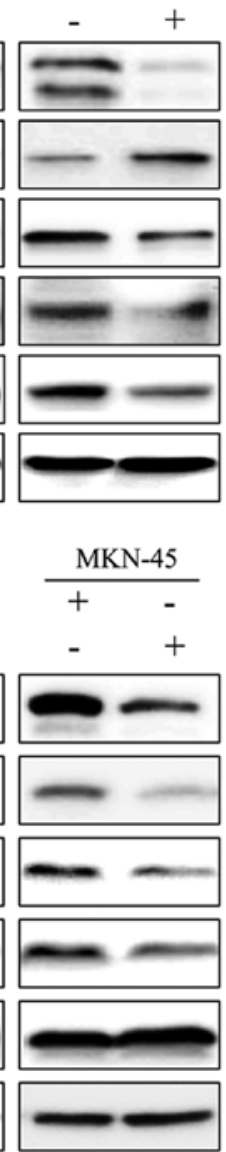

$\mathrm{B}$

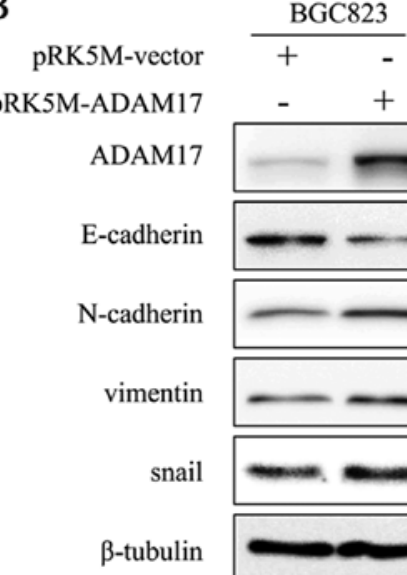

$\mathrm{D}$

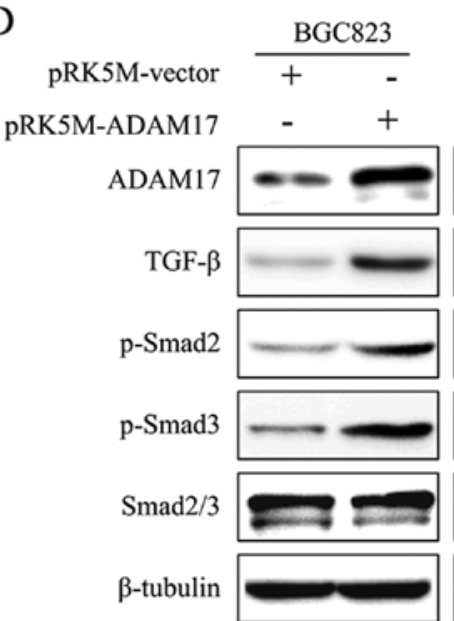

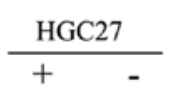
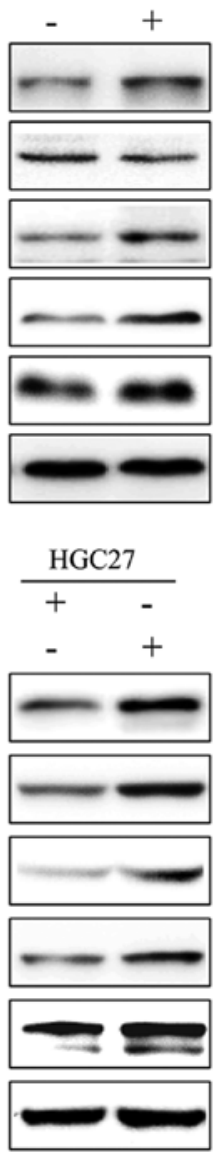

Figure 5. ADAM17 promotes epithelial-mesenchymal transition (EMT) via TGF- $\beta$ /Smad signaling in gastric carcinoma cells. (A) The protein levels of E-cadherin, N-cadherin, vimentin and Snail were measured by western blot analysis in MGC803 and MKN45 sh-ADAM17 stable infected cells. (B) After transfected with pRK5M-vector or pRK5M-ADAM17 plasmid in BGC823 and HGC27 cells, the protein levels of E-cadherin, N-cadherin, vimentin and Snail were measured by western blot analysis. (C) TGF- $\beta /$ Smad signaling pathway relative proteins were detected by western blot analysis in MGC803 and MKN45 sh-ADAM17 stable infected cells. (D) After transfected with pRK5M-vector or pRK5M-ADAM17 plasmid in BGC823 and HGC27 cells, TGF- 3 / Smad signaling pathway relative proteins were detected by western blot analysis.

and MKN45 cells expressing either sh-EGFP or sh-ADAM17. MGC803 and MKN45 cells expressing sh-ADAM17 displayed reduced motility in comparison to MGC803 and MKN45 cells expressing sh-EGFP (Fig. 3B). To confirm the above results, HGC27 cells and BGC823 cells were transfected with pRK5M-vector or pRK5M-ADAM17 plasmids. Conversely, overexpression of ADAM17 promoted the ability of migration in HGC27 and BGC823 cells (Fig. 3C and D). These data suggest that ADAM17 promotes the ability of migration in gastric carcinoma cells.

ADAM17 promotes cell invasion in gastric carcinoma cells. Then, we examined the effect of ADAM17 on the invasion ability of gastric carcinoma cells using BD Matrigel invasion assays. We transfected MGC803 and MKN45 cells with sh-EGFP or sh-ADAM17 plasmids, and HGC27 and BGC823 cells with pRK5M-vector or pRK5M-ADAM17 plasmids for $72 \mathrm{~h}$. The number of invasive cells were $170 \pm 12$ and $74 \pm 9$

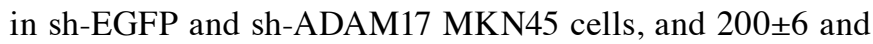
$98 \pm 8$ in sh-EGFP and sh-ADAM17 MGC803 cells (Fig. 4A), indicating that knockdown of ADAM17 obviously inhibited the invasion ability of MGC803 and MKN45 cells. Moreover, the number of invasive cells were $105 \pm 9$ and $200 \pm 5$ in vector and pRK5M-ADAM17 BGC823 cells, and 98 \pm 11 and $210 \pm 8$ in vector and pRK5M-ADAM17 HGC27 cells, suggesting that upregulation of ADAM17 significantly enhanced the invasion ability of BGC823 and HGC27 cells (Fig. 4C). Also, ADAM17 knockdown was conduced to downregulate the MMP-2 and MMP-9 at both mRNA and protein levels in MGC803 and MKN45 cells (Fig. 4B and E), while ADAM17 overexpression upregulated the expression of MMP-2 and MMP-9 in BGC823 andHGC27 cells (Fig. 4D and F). The above data suggest that ADAM17 promotes the invasion ability of gastric cancer cells.

ADAM17 promotes EMT via TGF- $\beta /$ Smad signaling in gastric carcinoma cells. The EMT is deemed to be associated with the ability of migration and invasion in cancer cells. Therefore, we detected EMT markers at the protein level by western blotting. Our data suggested that ADAM17 knockdown resulted in downregulation of vimentin, Snail, $\mathrm{N}$-cadherin and upregulation of E-cadherin in MGC803 and MKN45 cells (Fig. 5A). In contrast, ADAM17 overexpression led to upregulation of vimentin, Snail, N-cadherin and downregulation of E-cadherin in BGC823 and HGC27 cells 
(Fig. 5B). It is confirmed that ADAM17 promotes EMT in gastric carcinoma cells. As TGF- $\beta /$ Smad signaling is closely related to EMT in cancer, we investigated the effects of ADAM17 on the classic TGF- $\beta /$ Smad signaling. We found that ADAM17 knockdown downregulated TGF- $\beta$, p-Smad2/3 in MGC803 and MKN45 cells (Fig. 5C), while ADAM17 overexpression resulted in upregulation of TGF- $\beta$, p-Smad $2 / 3$ in BGC823 and HGC27 cells (Fig. 5D). Knocking down or overexpressing ADAM17 had no influence on total Smad2/3 protein. These data suggest that ADAM17 promotes EMT in gastric carcinoma cells via TGF- $\beta /$ Smad signaling.

\section{Discussion}

It has been reported that ADAM17 may function as an oncogene to promote cancer cell growth (18). ADAM17 expression is significantly increased in different types of cancers, including gastric cancer (19-22). In this study, we identify that ADAM17 promotes proliferation, migration and invasion in gastric cancer cells. Importantly, we found that ADAM17 promotes EMT probably via TGF- $\beta /$ Smad signaling pathway in gastric cancer. These findings suggest that ADAM17 could represent a novel anticancer strategy.

Epithelial-mesenchymal transition (EMT) is a critical cellular process in cancer metastasis, during which epithelial polarized cells become motile mesenchymal cells (23). The process of EMT consists of three major steps in cancer: i) loss of cell-cell junctions and a decrease in the epithelial marker E-cadherin; ii) acquisition of the mesenchymal marker $\mathrm{N}$-cadherin; and iii) cytoskeleton rearrangement intended for invasive properties. Additionally, these changes are paralleled with secretion of matrix metalloproteinase-2/-9 (MMP-2/-9) and focal adhesion kinase (FAK) (24). MMP-2/-9, proteolytic enzymes that degrade and modify the extracellular matrix (ECM), act directly on cell surface molecules and activate EMT (25). In the present study, we find that ADAM17 overexpression elevates the expression of MMP-2 and MMP-9, while ADAM17 knockdown downregulates the expression of MMP-2 and MMP-9. Therefore, we identified that ADAM17 elevates the expression of MMP-2 and MMP-9 thereby accelerating EMT.

It has been reported firmly that TGF- $\beta$ signaling pathway plays crucial roles in regulating malignancy initiation, progression and metastasis, including gastric cancer (26). The effects of TGF- $\beta$ on migration and invasion are associated with changes in ECM components, including collagen (27), fibronectin (28), laminin (29), MMP-2 and MMP-9 $(30,31)$. ADAM17 is also involved in proteolytical digestion of collagen IV of the ECM and the release from the cell surface of several integral proteins, which suggest that ADAM17 affects the invasive activity of a variety of cancers $(32,33)$. Therefore, there may be a possible link between ADAM17 and TGF- $\beta$. In this study, we presented clear evidence that ADAM17 induced expression of the TGF- $\beta$, and increased phosphorylated Smad2/3 while the total Smad2/3 expression was relatively unchanged. In the TGF- $\beta$ signaling pathway, TGF- $\beta$ receptor kinases phosphorylated Smad2 and Smad3 in the $\mathrm{C}$ terminal residue, resulting in forming a complex with Smad4, which plays the role of a common mediator, and the nuclear translocation to regulate gene expression leading to the stimulation of EMT $(34,35)$.
Hence, it is confirmed that ADAM17 promotes EMT probably via TGF- $\beta /$ Smad signaling in gastric carcinoma cells.

In conclusion, ADAM17 promotes proliferation, migration and invasion in gastric carcinoma cells. Importantly, the results detail a mechanism of ADAM17-mediated EMT through upregulating TGF- $\beta /$ Smad signaling pathway. These findings suggest that ADAM17 might be an important therapeutic target candidate in gastric cancer.

\section{Acknowledgements}

The present study was supported by grants from the National Natural Science Foundation of China (81472333 and 81372718) and the Natural Science Foundation of Jiangsu Province (BK20131247).

\section{References}

1. Jemal A, Center MM, DeSantis C and Ward EM: Global patterns of cancer incidence and mortality rates and trends. Cancer Epidemiol Biomarkers Prev 19: 1893-1907, 2010.

2. Liu Y, Feng Y, Gao Y and Hou R: Clinical benefits of combined chemotherapy with S-1, oxaliplatin, and docetaxel in advanced gastric cancer patients with palliative surgery. Onco Targets Ther 9: 1269-1273, 2016.

3. Pecqueux M, Fritzmann J, Adamu M, Thorlund K, Kahlert C, Reißfelder C, Weitz J and Rahbari NN: Free intraperitoneal tumor cells and outcome in gastric cancer patients: A systematic review and meta-analysis. Oncotarget 6: 35564-35578, 2015.

4. Xu P and Derynck R: Direct activation of TACE-mediated ectodomain shedding by p38 MAP kinase regulates EGF receptor-dependent cell proliferation. Mol Cell 37: 551-566, 2010.

5. Zhang TC, Zhu WG, Huang MD, Fan RH and Chen XF: Prognostic value of ADAM17 in human gastric cancer. Med Oncol 29: 2684-2690, 2012.

6. Kenny PA and Bissell MJ: Targeting TACE-dependent EGFR ligand shedding in breast cancer. J Clin Invest 117: 337-345, 2007.

7. Szalad A, Katakowski M, Zheng X, Jiang F and Chopp M: Transcription factor Sp1 induces ADAM17 and contributes to tumor cell invasiveness under hypoxia. J Exp Clin Cancer Res 28: 129, 2009.

8. Yoshimura T, Tomita T, Dixon MF, Axon AT, Robinson PA and Crabtree JE: ADAMs (a disintegrin and metalloproteinase) messenger RNA expression in Helicobacter pylori-infected, normal, and neoplastic gastric mucosa. J Infect Dis 185: 332-340, 2002.

9. Yasuda H, Hirata S, Inoue $\mathrm{K}$, Mashima H, Ohnishi $\mathrm{H}$ and Yoshiba M: Involvement of membrane-type bile acid receptor M-BAR/TGR5 in bile acid-induced activation of epidermal growth factor receptor and mitogen-activated protein kinases in gastric carcinoma cells. Biochem Biophys Res Commun 354: 154-159, 2007.

10. Ebi M, Kataoka H, Shimura T, Kubota E, Hirata Y, Mizushima T, Mizoshita T, Tanaka M, Mabuchi M, Tsukamoto H, et al: TGF $\beta$ induces proHB-EGF shedding and EGFR transactivation through ADAM activation in gastric cancer cells. Biochem Biophys Res Commun 402: 449-454, 2010.

11. Tape CJ, Willems SH, Dombernowsky SL, Stanley PL, Fogarasi M, Ouwehand W, McCafferty J and Murphy G: Crossdomain inhibition of TACE ectodomain. Proc Natl Acad Sci USA 108: 5578-5583, 2011.

12. Richards FM, Tape CJ, Jodrell DI and Murphy G: Anti-tumour effects of a specific anti-ADAM17 antibody in an ovarian cancer model in vivo. PLoS One 7: e40597, 2012.

13. Yang J and Weinberg RA: Epithelial-mesenchymal transition: At the crossroads of development and tumor metastasis. Dev Cell 14: 818-829, 2008.

14. Huang L, Wu RL and Xu AM: Epithelial-mesenchymal transition in gastric cancer. Am J Transl Res 7: 2141-2158, 2015.

15. Savagner P: Epithelial-mesenchymal transitions: From cell plasticity to concept elasticity. Curr Top Dev Biol 112: 273-300, 2015. 
16. Polyak K and Weinberg RA: Transitions between epithelial and mesenchymal states: Acquisition of malignant and stem cell traits. Nat Rev Cancer 9: 265-273, 2009.

17. Zheng $\mathrm{H}$ and Kang Y: Multilayer control of the EMT master regulators. Oncogene 33: 1755-1763, 2014.

18. Duffy MJ, Mullooly M, O'Donovan N, Sukor S, Crown J, Pierce A and McGowan PM: The ADAMs family of proteases: New biomarkers and therapeutic targets for cancer? Clin Proteomics 8: 9, 2011

19. Wu B, Sha L, Wang Y, Xu W, Yu Y, Feng F, Sun C and Xia L: Diagnostic and prognostic value of a disintegrin and metalloproteinase-17 in patients with gliomas. Oncol Lett 8: 2616-2620, 2014.

20. Cai M, Wang Z, Zhang J, Zhou H, Jin L, Bai R and Weng Y: Adam17, a target of mir-326, promotes EMT-induced cells invasion in lung adenocarcinoma. Cell Physiol Biochem 36: $1175-1185,2015$.

21. Van Schaeybroeck S, Kalimutho M, Dunne PD, Carson R, Allen W, Jithesh PV, Redmond KL, Sasazuki T, Shirasawa S, Blayney J, et al: ADAM17-dependent c-MET-STAT3 signaling mediates resistance to MEK inhibitors in KRAS mutant colorectal cancer. Cell Rep 7: 1940-1955, 2014.

22. Shou ZX, Jin $X$ and Zhao ZS: Upregulated expression of ADAM17 is a prognostic marker for patients with gastric cancer. Ann Surg 256: 1014-1022, 2012.

23. Tennakoon $\mathbf{A H}$, Izawa $\mathrm{T}$, Kuwamura $\mathbf{M}$ and Yamate $\mathbf{J}$ : Pathogenesis of type 2 epithelial to mesenchymal transition (EMT) in renal and hepatic fibrosis. J Clin Med 5: 5, 2015.

24. Kim YJ, Choi WI, Jeon BN, Choi KC, Kim K, Kim TJ, Ham J, Jang HJ, Kang KS and Ko H: Stereospecific effects of ginsenoside $20-\mathrm{Rg} 3$ inhibits TGF- $\beta 1$-induced epithelial-mesenchymal transition and suppresses lung cancer migration, invasion and anoikis resistance. Toxicology 322: 23-33, 2014

25. Cichon MA and Radisky DC: ROS-induced epithelial-mesenchymal transition in mammary epithelial cells is mediated by $\mathrm{NF}-\kappa \mathrm{B}-$ dependent activation of Snail. Oncotarget 5: 2827-2838, 2014.

26. Zhou Q, Zheng X, Chen L, Xu B, Yang X, Jiang J and Wu C: Smad2/3/4 pathway contributes to TGF-beta-induced MiRNA$181 \mathrm{~b}$ Expression to promote gastric cancer metastasis by targeting Timp3. Cell Physiol Biochem 39: 453-466, 2016.
27. Zimmerman KA, Xing D, Pallero MA, Lu A, Ikawa M, Black L, Hoyt KL, Kabarowski JH, Michalak M and Murphy-Ullrich JE: Calreticulin regulates neointima formation and collagen deposition following carotid artery ligation. J Vasc Res 52: 306-320, 2015.

28. Huang P, Zhang Y, Jiang T and Zhang N: Effects of p38 MAPK signaling pathway and aldose reductase on transforming growth factor-beta1 induced expression of fibronectin in cultured human mesangial cells. Zhonghua Bing Li Xue Za Zhi 44: 778-782, 2015 (In Chinese)

29. Tennant BR, Chen J, Shih AZ, Luciani DS and Hoffman BG: Myt 3 mediates laminin-V/integrin- $\beta 1$-induced islet-cell migration via Tgfbi. Mol Endocrinol 29: 1254-1268, 2015.

30. Hirunsai M, Srikuea R and Yimlamai T: Heat stress promotes extracellular matrix remodelling via TGF-betal and MMP-2/ TIMP-2 modulation in tenotomised soleus and plantaris muscles. Int J Hyperthermia 31: 336-348, 2015.

31. Zhao J, Cheng Q, Ye P, Yang G, Liu S, Ao Q, Liu Y and Hu Y: Atorvastatin improves pathological changes in the aged kidney by upregulating peroxisome proliferator-activated receptor expression and reducing matrix metalloproteinase- 9 and transforming growth factor- $\beta 1$ levels. Exp Gerontol 74: 37-42, 2016.

32. Weskamp G, Mendelson K, Swendeman S, Le Gall S, Ma Y, Lyman S, Hinoki A, Eguchi S, Guaiquil V, Horiuchi K, et al: Pathological neovascularization is reduced by inactivation of ADAM17 in endothelial cells but not in pericytes. Circ Res 106: 932-940, 2010

33. Lu Y, Jiang F, Zheng X, Katakowski M, Buller B, To SS and Chopp M: TGF- $\beta 1$ promotes motility and invasiveness of glioma cells through activation of ADAM17. Oncol Rep 25: 1329-1335, 2011.

34. Song J: EMT or apoptosis: A decision for TGF-beta. Cell Res 17: 289-290, 2007.

35. Liang Y, Zhu F, Zhang H, Chen D, Zhang X, Gao Q and Li Y: Conditional ablation of TGF- $\beta$ signaling inhibits tumor progression and invasion in an induced mouse bladder cancer model. Sci Rep 6: 29479, 2016. 